\title{
Vector spherical wavefunctions for orthorhombic dielectric-magnetic material with gyrotropic-like magnetoelectric properties
}

\author{
Akhlesh Lakhtakia $^{1 *}$ and Tom G. Mackay ${ }^{1,2}$ \\ ${ }^{1}$ NanoMM - Nanoengineered Metamaterials Group, Department of Engineering Science and Mechanics, \\ Pennsylvania State University, University Park, PA 16802-6812, USA \\ ${ }^{2}$ School of Mathematics and Maxwell Institute for Mathematical Sciences, \\ University of Edinburgh, Edinburgh EH9 3JZ, United Kingdom
}

\begin{abstract}
Vector spherical wavefunctions were derived in closed-form to represent time-harmonic electromagnetic fields in an orthorhombic dielectric-magnetic material with gyrotropic-like magnetoelectric properties. These wavefunctions were used to formulate the $T$ matrix for scattering by a three-dimensional object composed of the chosen material. Furthermore, a closed-form, coordinate-free expression of the dyadic Green function for the chosen material was derived. Expressions ascertained for the singularity behavior will be useful for formulating volume integral equations for scattering inside the chosen material. A bilinear expansion of the dyadic Green function was obtained in terms of the derived vector spherical wavefunctions.
\end{abstract}

\section{Introduction}

Closed-form solutions of the vector differential equation

$$
\nabla \times[\nabla \times \mathbf{F}(\mathbf{r})]-\kappa^{2} \mathbf{F}(\mathbf{r})=\mathbf{0}
$$

in a spherical coordinate system to represent the field $\mathbf{F}$ and the position vector $\mathbf{r}$ can be traced back to as early as the 1860s, when Clebsch considered the scattering of an elastodynamic wave by a solid sphere, as recounted by Logan in 1965 [1]. In frequency-domain electromagnetics for homogeneous, isotropic, dielectric materials, this equation simplifies to the vector Helmholtz equation

$$
\nabla^{2} \mathbf{F}(\mathbf{r})+\kappa^{2} \mathbf{F}(\mathbf{r})=\mathbf{0}
$$

because of the constraint $\nabla \cdot \mathbf{F}(\mathbf{r})=0$. Credit for this reduction and application to the optical scattering response of a homogeneous, isotropic, dielectric sphere evidently goes to a series of papers by Ludvig Lorenz from the second half of the 19th century [1,2]. However, Lorenz's 1880 paper [3-5] has largely been superseded in popular memory by the 1908 paper of Gustav Mie [6], most probably because Lorenz used a pre-Maxwellian theory of light whereas Mie used the frequency-domain Maxwell equations and also applied his solution to the very practical problem of scattering of light by colloidal spheres.

Solutions of Eqs. (1) and (2) in spherical coordinates are called vector spherical wavefunctions. For homogeneous, isotropic, dielectric materials, these wavefunctions involve $\kappa=k_{0} \sqrt{\epsilon_{r}}$, where $k_{0}$ is the

\footnotetext{
${ }^{*}$ Corresponding author. E-mail: akhlesh@psu.edu
} 
free-space wavenumber and $\epsilon_{r}$ is the (frequency-dependent) relative permittivity scalar. Extension to homogeneous, isotropic, dielectric-magnetic materials $[7,8]$ is straightforward with the vector Helmholtz equation requiring only a simple redefinition: $\kappa=k_{0} \sqrt{\epsilon_{r} \mu_{r}}$, where $\mu_{r}$ is the relative permeability scalar, also frequency dependent. Extension to homogeneous bi-isotropic materials, which require the solution of

$$
\nabla \times[\nabla \times \mathbf{F}(\mathbf{r})]+i k_{0}(\zeta-\xi) \nabla \times \mathbf{F}(\mathbf{r})-k_{0}^{2}\left(\epsilon_{r} \mu_{r}-\zeta \xi\right) \mathbf{F}(\mathbf{r})=\mathbf{0}
$$

with $\xi$ and $\zeta$ as frequency-dependent magnetoelectric scalars, came much later [9]; even so, the solutions of Eq. (3) are simple combinations of the solutions of Eq. (2) $[9,10]$.

Vector spherical wavefunctions for homogeneous anisotropic materials have not been found in closed form. A synthetic approach that requires finding vector cartesian wavefunctions [11] and subsequent transformation to spherical coordinates [12] does not yield closed-form expressions for the vector spherical wavefunctions $[13,14]$. This is because a spherical coordinate system is generally incompatible with the cartesian nature of anisotropy.

An exception is furnished by the Minkowski constitutive relations of a simply moving material that is an isotropic dielectric-magnetic material in its co-moving frame of reference. These constitutive relations can be stated as

$$
\begin{aligned}
& \mathbf{D}(\mathbf{r})=\epsilon_{0} \epsilon_{r}[\gamma \underline{\underline{I}}+(1-\gamma) \hat{\mathbf{v}} \hat{\mathbf{v}}] \cdot \mathbf{E}(\mathbf{r})+\beta \hat{\mathbf{v}} \times \mathbf{H}(\mathbf{r}) \\
& \mathbf{B}(\mathbf{r})=\mu_{0} \mu_{r}[\gamma \underline{\underline{I}}+(1-\gamma) \hat{\mathbf{v}} \hat{\mathbf{v}}] \cdot \mathbf{H}(\mathbf{r})-\beta \hat{\mathbf{v}} \times \mathbf{E}(\mathbf{r})
\end{aligned}
$$

where $\epsilon_{0}$ and $\mu_{0}$ are, respectively, the permittivity and the permeability of free space; $\epsilon_{r}, \mu_{r}, \gamma$, and $\beta$ are real-valued scalars; the unit vector $\hat{\mathbf{v}}$ is parallel to the velocity of the material; and $\underline{\underline{I}}$ is the identity dyadic. By a combination of two transformations [15], the frequency-domain Maxwell equations in this material can be converted to frequency-domain Maxwell equations in a homogeneous, isotropic, dielectricmagnetic material [16]. The same algebraic manipulation is possible for a material whose constitutive relations are inspired by those of gravitationally affected vacuum and which can potentially be fabricated by properly dispersing electrically small bent-wire and other complex inclusions of different shapes and materials in some host materials [15].

We show in this paper that a similar combination of transformations can be fruitfully applied to the more general material described by the constitutive relations

$$
\begin{aligned}
& \mathbf{D}(\mathbf{r})=\epsilon_{0} \epsilon_{r} \underline{\underline{S}} \cdot \underline{\underline{A}} \cdot \underline{\underline{A}} \cdot \underline{\underline{S}}^{-1} \cdot \mathbf{E}(\mathbf{r})-c_{0}^{-1} \boldsymbol{\Gamma} \times \mathbf{H}(\mathbf{r}), \\
& \mathbf{B}(\mathbf{r})=\mu_{0} \mu_{r} \underline{\underline{S}} \cdot \underline{\underline{A}} \cdot \underline{\underline{A}} \cdot \underline{\underline{S}}^{-1} \cdot \mathbf{H}(\mathbf{r})+c_{0}^{-1} \boldsymbol{\Gamma} \times \mathbf{E}(\mathbf{r}) .
\end{aligned}
$$

In these equations, $c_{0}=1 / \sqrt{\epsilon_{0} \mu_{0}}$ is the speed of light in free space; $\epsilon_{r} \neq 0$ and $\mu_{r} \neq 0$ are complexvalued scalars; $\underline{\underline{S}}$ is either a rotation dyadic or a product of rotation dyadics; the diagonal dyadic $\underline{\underline{A}}$ is positive definite with real-valued elements; and the gyrotropic vector $\boldsymbol{\Gamma}$ can have complex-valued components. For later use, we define the free-space impedance $\eta_{0}=\sqrt{\mu_{0} / \epsilon_{0}}$ and the relative impedance $\eta_{r}(\omega)=\sqrt{\mu_{r}(\omega) / \epsilon_{r}(\omega)}$. The dependences of the constitutive variables $\epsilon_{r}, \mu_{r}, \underline{\underline{A}}$, and $\boldsymbol{\Gamma}$ on the angular frequency $\omega>0$ have not been mentioned for notational compactness, and an $\exp (-i \omega t)$ dependence on time $t$ is implicit.

Accordingly, using a set of three transformations developed in Sec. 2, we determine closed-form expressions for vector spherical wavefunctions applicable to a material with the constitutive relations (6) and (7), as shown in Sec. 3. The derived vector spherical wavefunctions are used in Sec. 3.3 to formulate the $\mathrm{T}$ matrix for scattering by a three-dimensional object composed of the chosen material. Section 4 is devoted to the radiation field of an electric source current density distribution in the embedded material: a closed-form coordinate-free expression of the dyadic Green function is obtained, followed by the elucidation of its singularity behavior and its representation in a bilinear form involving the derived vector spherical wavefunctions. 


\section{Transformations}

Representing the position vector $\mathbf{r} \equiv(x, y, z) \equiv(r, \theta, \phi)$, and denoting the source current density by $\mathbf{J}(\mathbf{r})$, we aim to solve

$$
\begin{aligned}
& \nabla \times \mathbf{E}(\mathbf{r})=i \omega \mathbf{B}(\mathbf{r}), \\
& \nabla \times \mathbf{H}(\mathbf{r})=-i \omega \mathbf{D}(\mathbf{r})+\mathbf{J}(\mathbf{r}),
\end{aligned}
$$

when Eqs. (6) and (7) have been substituted therein. This is done after applying three transformations in succession so that we have to deal with effectively a homogeneous, isotropic, dielectric-magnetic material.

\section{$2.1 \quad$ First transformation}

Defining the auxiliary fields [17]

$$
\begin{aligned}
& \mathbf{e}(\mathbf{r})=\mathbf{E}(\mathbf{r}) \exp \left(-i k_{0} \boldsymbol{\Gamma} \cdot \mathbf{r}\right) \\
& \mathbf{h}(\mathbf{r})=\mathbf{H}(\mathbf{r}) \exp \left(-i k_{0} \boldsymbol{\Gamma} \cdot \mathbf{r}\right),
\end{aligned}
$$

and auxiliary source current density

$$
\mathbf{j}(\mathbf{r})=\mathbf{J}(\mathbf{r}) \exp \left(-i k_{0} \boldsymbol{\Gamma} \cdot \mathbf{r}\right),
$$

and noting that $k_{0}=\omega / c_{0}$, we transform Eqs. (8) and (9) to

$$
\begin{aligned}
& \nabla \times \mathbf{e}(\mathbf{r})=i \omega \mu_{0} \mu_{r} \underline{\underline{S}} \cdot \underline{\underline{A}} \cdot \underline{\underline{A}} \cdot \underline{\underline{S}}^{-1} \cdot \mathbf{h}(\mathbf{r}) \\
& \nabla \times \mathbf{h}(\mathbf{r})=-i \omega \epsilon_{0} \epsilon_{r} \underline{\underline{S}} \cdot \underline{\underline{A}} \cdot \underline{\underline{A}} \cdot \underline{\underline{S}}^{-1} \cdot \mathbf{e}(\mathbf{r})+\mathbf{j}(\mathbf{r}) .
\end{aligned}
$$

\subsection{Second transformation}

Next, we define another set of auxiliary quantities

$$
\begin{aligned}
& \mathbf{e}^{\prime}\left(\mathbf{r}^{\prime}\right)=\underline{S}^{-1} \cdot \mathbf{e}(\mathbf{r}), \\
& \mathbf{h}^{\prime}\left(\mathbf{r}^{\prime}\right)=\underline{S}^{-1} \cdot \mathbf{h}(\mathbf{r}), \\
& \mathbf{j}^{\prime}\left(\mathbf{r}^{\prime}\right)=\underline{S}^{-1} \cdot \mathbf{j}(\mathbf{r}),
\end{aligned}
$$

and coordinates $\mathbf{r}^{\prime} \equiv\left(x^{\prime}, y^{\prime}, z^{\prime}\right) \equiv\left(r^{\prime}, \theta^{\prime}, \phi^{\prime}\right)$ such that

$$
\mathbf{r}^{\prime}=\underline{\underline{S}} \cdot \mathbf{r}
$$

in order to transform Eqs. (13) and (14) to

$$
\begin{aligned}
& \nabla^{\prime} \times \mathbf{e}^{\prime}\left(\mathbf{r}^{\prime}\right)=i \omega \mu_{0} \mu_{r} \underline{\underline{A}} \cdot \underline{\underline{A}} \cdot \mathbf{h}^{\prime}\left(\mathbf{r}^{\prime}\right), \\
& \nabla^{\prime} \times \mathbf{h}^{\prime}\left(\mathbf{r}^{\prime}\right)=-i \omega \epsilon_{0} \epsilon_{r} \underline{\underline{A}} \cdot \underline{\underline{A}} \cdot \mathbf{e}^{\prime}\left(\mathbf{r}^{\prime}\right)+\mathbf{j}^{\prime}\left(\mathbf{r}^{\prime}\right),
\end{aligned}
$$

because

$$
\underline{\underline{S}}^{-1} \cdot(\nabla \times \underline{\underline{I}}) \cdot \underline{\underline{S}}=\nabla^{\prime} \times \underline{\underline{I}} .
$$

This transformation merely amounts to a rigid rotation of the coordinate system, and can be avoided by an appropriate choice of the $(x, y, z)$ coordinate system. 


\subsection{Third transformation}

Finally, we make use of an affine transformation $[18,19]$ associated with the scaling of space as

$$
\tilde{\mathbf{r}}=\underline{\underline{A}} \cdot \mathbf{r}^{\prime}
$$

where $\tilde{\mathbf{r}} \equiv(\tilde{x}, \tilde{y}, \tilde{z}) \equiv(\tilde{r}, \tilde{\theta}, \tilde{\phi})$, and define the final set of auxiliary quantities as

$$
\begin{aligned}
& \tilde{\mathbf{e}}\left(\mathbf{r}^{\prime}\right)=\underline{\underline{A}} \cdot \mathbf{e}^{\prime}(\tilde{\mathbf{r}}), \\
& \tilde{\mathbf{h}}\left(\mathbf{r}^{\prime}\right)=\underline{\underline{A}} \cdot \mathbf{h}^{\prime}(\tilde{\mathbf{r}}), \\
& \tilde{\mathbf{j}}\left(\mathbf{r}^{\prime}\right)=(\operatorname{adj} \underline{\underline{A}}) \cdot \mathbf{j}^{\prime}(\tilde{\mathbf{r}}),
\end{aligned}
$$

to obtain

$$
\begin{aligned}
& \nabla^{\prime} \times \tilde{\mathbf{e}}\left(\mathbf{r}^{\prime}\right)=i \omega \mu_{0} \mu_{r} A \tilde{\mathbf{h}}\left(\mathbf{r}^{\prime}\right), \\
& \nabla^{\prime} \times \tilde{\mathbf{h}}\left(\mathbf{r}^{\prime}\right)=-i \omega \epsilon_{0} \epsilon_{r} A \tilde{\mathbf{e}}\left(\mathbf{r}^{\prime}\right)+\tilde{\mathbf{j}}\left(\mathbf{r}^{\prime}\right),
\end{aligned}
$$

where $A$ is the determinant of $\underline{\underline{A}}$. Equations (26) and (27) are identical to analogous equations for a homogeneous, isotropic, dielectric-magnetic material.

From these two equations, we get

$$
\begin{aligned}
& \nabla^{\prime} \times\left[\nabla^{\prime} \times \tilde{\mathbf{e}}\left(\mathbf{r}^{\prime}\right)\right]-k_{0}^{2} \epsilon_{r} \mu_{r} A^{2} \tilde{\mathbf{e}}\left(\mathbf{r}^{\prime}\right)=i \omega \mu_{0} \mu_{r} A \tilde{\mathbf{j}}\left(\mathbf{r}^{\prime}\right), \\
& \nabla^{\prime} \times\left[\nabla^{\prime} \times \tilde{\mathbf{h}}\left(\mathbf{r}^{\prime}\right)\right]-k_{0}^{2} \epsilon_{r} \mu_{r} A^{2} \tilde{\mathbf{h}}\left(\mathbf{r}^{\prime}\right)=\nabla^{\prime} \times \tilde{\mathbf{j}}\left(\mathbf{r}^{\prime}\right) .
\end{aligned}
$$

In the absence of a source current density, these equations are the same as Eq. (1).

\section{Field representation in a source-free region}

Homogeneous solutions of the Eqs. (28) and (29) in the spherical coordinate system were obtained by Clebsch [1]. Thus, in the absence of the source current density,

$$
\tilde{\mathbf{e}}\left(\mathbf{r}^{\prime}\right)=\sum_{j=1,3} \sum_{s=e, 0} \sum_{n=0}^{\infty} \sum_{m=0}^{n}\left[a_{s m n}^{(j)} \mathbf{L}_{s m n}^{(j)}\left(k \mathbf{r}^{\prime}\right)+b_{s m n}^{(j)} \mathbf{M}_{s m n}^{(j)}\left(k \mathbf{r}^{\prime}\right)+c_{s m n}^{(j)} \mathbf{N}_{s m n}^{(j)}\left(k \mathbf{r}^{\prime}\right)\right]
$$

and

$$
\tilde{\mathbf{h}}\left(\mathbf{r}^{\prime}\right)=\sum_{j=1,3} \sum_{s=e, o} \sum_{n=0}^{\infty} \sum_{m=0}^{n}\left[\alpha_{s m n}^{(j)} \mathbf{L}_{s m n}^{(j)}\left(k \mathbf{r}^{\prime}\right)+\beta_{s m n}^{(j)} \mathbf{M}_{s m n}^{(j)}\left(k \mathbf{r}^{\prime}\right)+\gamma_{s m n}^{(j)} \mathbf{N}_{s m n}^{(j)}\left(k \mathbf{r}^{\prime}\right)\right],
$$

where the wavenumber

$$
k=k_{0} A \sqrt{\epsilon_{r}} \sqrt{\mu_{r}} ;
$$

the standard vector spherical wave functions [20]

$$
\begin{aligned}
& \mathbf{L}_{s m n}^{(1)}\left(k \mathbf{r}^{\prime}\right)=\frac{1}{k} \nabla^{\prime}\left[Y_{s m n}\left(\theta^{\prime}, \phi^{\prime}\right) j_{n}\left(k r^{\prime}\right)\right] \\
& \mathbf{L}_{s m n}^{(3)}\left(k \mathbf{r}^{\prime}\right)=\frac{1}{k} \nabla^{\prime}\left[Y_{s m n}\left(\theta^{\prime}, \phi^{\prime}\right) h_{n}^{(1)}\left(k r^{\prime}\right)\right] \\
& \mathbf{M}_{s m n}^{(1)}\left(k \mathbf{r}^{\prime}\right)=\nabla^{\prime} \times\left[\mathbf{r}^{\prime} Y_{s m n}\left(\theta^{\prime}, \phi^{\prime}\right) j_{n}\left(k r^{\prime}\right)\right] \\
& \mathbf{M}_{s m n}^{(3)}\left(k \mathbf{r}^{\prime}\right)=\nabla^{\prime} \times\left[\mathbf{r}^{\prime} Y_{s m n}\left(\theta^{\prime}, \phi^{\prime}\right) h_{n}^{(1)}\left(k r^{\prime}\right)\right], \\
& \mathbf{N}_{s m n}^{(j)}\left(k \mathbf{r}^{\prime}\right)=\frac{1}{k} \nabla^{\prime} \times \mathbf{M}_{s m n}^{(j)}\left(k \mathbf{r}^{\prime}\right)
\end{aligned}
$$


the tesseral harmonics

$$
\begin{aligned}
& Y_{\text {emn }}\left(\theta^{\prime}, \phi^{\prime}\right)=P_{n}^{m}\left(\cos \theta^{\prime}\right) \cos \left(m \phi^{\prime}\right), \\
& Y_{\text {omn }}\left(\theta^{\prime}, \phi^{\prime}\right)=P_{n}^{m}\left(\cos \theta^{\prime}\right) \sin \left(m \phi^{\prime}\right) ;
\end{aligned}
$$

$j_{n}(\bullet)$ is the spherical Bessel function of order $n, h_{n}^{(1)}(\bullet)$ is the spherical Hankel function of the first kind and order $n, P_{n}^{m}(\bullet)$ is the associated Legendre function of order $n$ and degree $m$; and the coefficients

$a_{s m n}^{(j)}, b_{s m n}^{(j)}, c_{s m n}^{(j)}, \alpha_{s m n}^{(j)}, \beta_{s m n}^{(j)}$, and $\gamma_{s m n}^{(j)}$ have to be determined from various stipulations on the spatial variations of $\tilde{\mathbf{e}}\left(\mathbf{r}^{\prime}\right)$ and $\tilde{\mathbf{h}}\left(\mathbf{r}^{\prime}\right)$.

As the identities $\nabla^{\prime} \times \mathbf{L}_{s m n}^{(j)}\left(k \mathbf{r}^{\prime}\right) \equiv \mathbf{0}, \nabla^{\prime} \cdot \mathbf{M}_{s m n}^{(j)}\left(k \mathbf{r}^{\prime}\right) \equiv 0$, and $\nabla^{\prime} \cdot \mathbf{N}_{s m n}^{(j)}\left(k \mathbf{r}^{\prime}\right) \equiv 0$ follow from the definitions (33)-(37), substitution of Eqs. (30) and (31) in Eqs. (26) and (27) yields the simplifications

$$
a_{s m n}^{(j)} \equiv 0, \quad \alpha_{s m n}^{(j)} \equiv 0 .
$$

Furthermore, as $\nabla^{\prime} \times \mathbf{M}_{s m n}^{(j)}\left(k \mathbf{r}^{\prime}\right)=k \mathbf{N}_{s m n}^{(j)}\left(k \mathbf{r}^{\prime}\right)$ and $\nabla^{\prime} \times \mathbf{N}_{s m n}^{(j)}\left(k \mathbf{r}^{\prime}\right)=k \mathbf{M}_{s m n}^{(j)}\left(k \mathbf{r}^{\prime}\right)$, the same substitution also yields the simplifications

$$
\beta_{s m n}^{(j)}=-\frac{i c_{s m n}^{(j)}}{\eta_{0} \eta_{r}(\omega)}, \quad \gamma_{s m n}^{(j)}=-\frac{i b_{s m n}^{(j)}}{\eta_{0} \eta_{r}(\omega)} .
$$

Accordingly, Eqs. (30) and (31) can be rewritten as follows:

$$
\begin{aligned}
\tilde{\mathbf{e}}\left(\mathbf{r}^{\prime}\right) & =\sum_{j=1,3} \sum_{s=e, o} \sum_{n=1}^{\infty} \sum_{m=0}^{n}\left[b_{s m n}^{(j)} \mathbf{M}_{s m n}^{(j)}\left(k \mathbf{r}^{\prime}\right)+c_{s m n}^{(j)} \mathbf{N}_{s m n}^{(j)}\left(k \mathbf{r}^{\prime}\right)\right] \\
\tilde{\mathbf{h}}\left(\mathbf{r}^{\prime}\right) & =-\frac{i}{\eta_{0} \eta_{r}(\omega)} \sum_{j=1,3} \sum_{s=e, o} \sum_{n=1}^{\infty} \sum_{m=0}^{n}\left[c_{s m n}^{(j)} \mathbf{M}_{s m n}^{(j)}\left(k \mathbf{r}^{\prime}\right)+b_{s m n}^{(j)} \mathbf{N}_{s m n}^{(j)}\left(k \mathbf{r}^{\prime}\right)\right] .
\end{aligned}
$$

Let us note here that the set of functions $\mathbf{M}_{s m n}^{(j)}\left(k \mathbf{r}^{\prime}\right)$ and $\mathbf{N}_{s m n}^{(j)}\left(k \mathbf{r}^{\prime}\right)$ is complete [21].

\subsection{Vector spherical wavefunctions}

Inverting the relations (23) and (24), we get

$$
\begin{aligned}
\mathbf{e}^{\prime}\left(\mathbf{r}^{\prime}\right)=\underline{\underline{A}}^{-1} \cdot \sum_{j=1,3} \sum_{s=e, o} \sum_{n=1}^{\infty} \sum_{m=0}^{n}\left[b_{s m n}^{(j)} \mathbf{M}_{s m n}^{(j)}\left(k \underline{\underline{A}}^{-1} \cdot \mathbf{r}^{\prime}\right)+c_{s m n}^{(j)} \mathbf{N}_{s m n}^{(j)}\left(k \underline{\underline{A}}^{-1} \cdot \mathbf{r}^{\prime}\right)\right], \\
\mathbf{h}^{\prime}\left(\mathbf{r}^{\prime}\right)=-\frac{i}{\eta_{0} \eta_{r}} \underline{\underline{A}}^{-1} \cdot \sum_{j=1,3} \sum_{s=e, o} \sum_{n=1}^{\infty} \sum_{m=0}^{n}\left[c_{s m n}^{(j)} \mathbf{M}_{s m n}^{(j)}\left(k \underline{\underline{A}}^{-1} \cdot \mathbf{r}^{\prime}\right)\right. \\
\left.+b_{s m n}^{(j)} \mathbf{N}_{s m n}^{(j)}\left(k \underline{\underline{A}}^{-1} \cdot \mathbf{r}^{\prime}\right)\right]
\end{aligned}
$$

from Eqs. (42) and (43). Next, an inversion involving Eqs. (15) and (16) yields

$$
\begin{array}{r}
\mathbf{e}(\mathbf{r})=\underline{\underline{S}} \cdot \underline{\underline{A}}^{-1} \cdot \sum_{j=1,3} \sum_{s=e, o} \sum_{n=1}^{\infty} \sum_{m=0}^{n}\left[b_{s m n}^{(j)} \mathbf{M}_{s m n}^{(j)}\left(k \underline{\underline{A}}^{-1} \cdot \underline{\underline{S}} \cdot \mathbf{r}\right)\right. \\
\left.+c_{s m n}^{(j)} \mathbf{N}_{s m n}^{(j)}\left(k \underline{\underline{A}}^{-1} \cdot \underline{\underline{S}} \cdot \mathbf{r}\right)\right] \\
\mathbf{h}(\mathbf{r})=-\frac{i}{\eta_{0} \eta_{r}} \underline{\underline{S}} \cdot \underline{\underline{A}}^{-1} \cdot \sum_{j=1,3} \sum_{s=e, o} \sum_{n=1}^{\infty} \sum_{m=0}^{n}\left[c_{s m n}^{(j)} \mathbf{M}_{s m n}^{(j)}\left(k \underline{\underline{A}}^{-1} \cdot \underline{\underline{S}} \cdot \mathbf{r}\right)\right. \\
\left.+b_{s m n}^{(j)} \mathbf{N}_{s m n}^{(j)}\left(k \underline{\underline{A}}^{-1} \cdot \underline{\underline{S}} \cdot \mathbf{r}\right)\right]
\end{array}
$$


from where we finally obtain

$$
\begin{aligned}
& \mathbf{E}(\mathbf{r})=\underline{\underline{S}} \cdot \underline{\underline{A}}^{-1} \cdot \sum_{j=1,3} \sum_{s=e, o} \sum_{n=1}^{\infty} \sum_{m=0}^{n}\left[b_{s m n}^{(j)} \mathbf{M}_{s m n}^{(j)}\left(k \underline{\underline{A}}^{-1} \cdot \underline{\underline{S}} \cdot \mathbf{r}\right)\right. \\
&\left.+c_{s m n}^{(j)} \mathbf{N}_{s m n}^{(j)}\left(k \underline{\underline{A}}^{-1} \cdot \underline{\underline{S}} \cdot \mathbf{r}\right)\right] \exp \left(i k_{0} \mathbf{\Gamma} \cdot \mathbf{r}\right), \\
& \mathbf{H}(\mathbf{r})=-\frac{i}{\eta_{0} \eta_{r}} \underline{\underline{S}} \cdot \underline{\underline{A}}^{-1} \cdot \sum_{j=1,3} \sum_{s=e, o} \sum_{n=1}^{\infty} \sum_{m=0}^{n}\left[c_{s m n}^{(j)} \mathbf{M}_{s m n}^{(j)}\left(k \underline{\underline{A}}^{-1} \cdot \underline{\underline{S}} \cdot \mathbf{r}\right)\right. \\
&\left.+b_{s m n}^{(j)} \mathbf{N}_{s m n}^{(j)}\left(k \underline{\underline{A}}^{-1} \cdot \underline{\underline{S}} \cdot \mathbf{r}\right)\right] \exp \left(i k_{0} \mathbf{\Gamma} \cdot \mathbf{r}\right) .
\end{aligned}
$$

Thus, for the material described by Eqs. (6) and (7), the desired vector spherical wavefunctions in the spherical coordinates $(r, \theta, \phi)$ are

$$
\mathbf{m}_{s m n}^{(j)}(\mathbf{r})=\exp \left(i k_{0} \boldsymbol{\Gamma} \cdot \mathbf{r}\right) \underline{\underline{S}} \cdot \underline{\underline{A}}^{-1} \cdot \mathbf{M}_{s m n}^{(j)}\left(k \underline{\underline{A}}^{-1} \cdot \underline{\underline{S}} \cdot \mathbf{r}\right)
$$

and

$$
\mathbf{n}_{s m n}^{(j)}(\mathbf{r})=\exp \left(i k_{0} \mathbf{\Gamma} \cdot \mathbf{r}\right) \underline{\underline{S}} \cdot \underline{\underline{A}}^{-1} \cdot \mathbf{N}_{s m n}^{(j)}\left(k \underline{\underline{A}}^{-1} \cdot \underline{\underline{S}} \cdot \mathbf{r}\right)
$$

Equations (48) and (49) can then be compactly written as

$$
\begin{aligned}
& \mathbf{E}(\mathbf{r})=\sum_{j=1,3} \sum_{s=e, o} \sum_{n=1}^{\infty} \sum_{m=0}^{n}\left[b_{s m n}^{(j)} \mathbf{m}_{s m n}^{(j)}(\mathbf{r})+c_{s m n}^{(j)} \mathbf{n}_{s m n}^{(j)}(\mathbf{r})\right] \\
& \mathbf{H}(\mathbf{r})=-\frac{i}{\eta_{0} \eta_{r}} \sum_{j=1,3} \sum_{s=e, o} \sum_{n=1}^{\infty} \sum_{m=0}^{n}\left[c_{s m n}^{(j)} \mathbf{m}_{s m n}^{(j)}(\mathbf{r})+b_{s m n}^{(j)} \mathbf{n}_{s m n}^{(j)}(\mathbf{r})\right] .
\end{aligned}
$$

The completeness of the set of functions $\mathbf{m}_{s m n}^{(j)}(\mathbf{r})$ and $\mathbf{n}_{s m n}^{(j)}(\mathbf{r})$ follows from the completeness of the set of functions $\mathbf{M}_{s m n}^{(j)}\left(k \mathbf{r}^{\prime}\right)$ and $\mathbf{N}_{s m n}^{(j)}\left(k \mathbf{r}^{\prime}\right)[21]$.

\subsection{Closed-form expressions}

Without any loss of generality, we set

$$
\left.\left.\underline{\underline{\underline{A}}}=\underline{\underline{\underline{I}}}=\underline{\alpha_{x}^{-1} \hat{\mathbf{x}} \hat{\mathbf{x}}+\alpha_{y}^{-1} \hat{\mathbf{y}} \hat{\mathbf{y}}+\hat{\mathbf{z}} \hat{\mathbf{z}}}\right\}\right\}
$$

where $\alpha_{x}>0$ and $\alpha_{y}>0$. In order to avoid extremely cumbersome expressions for the vector spherical wavefunctions $\mathbf{m}_{s m n}^{(j)}(\mathbf{r})$ and $\mathbf{n}_{s m n}^{(j)}(\mathbf{r})$, we also define wavefunctions $\mathcal{M}_{s m n}^{(j)}(\mathbf{r})$ and $\mathcal{N}_{s m n}^{(j)}(\mathbf{r})$ via

$$
\begin{aligned}
& \mathbf{m}_{s m n}^{(j)}(\mathbf{r})=\underline{\underline{A}}^{-1} \cdot \mathcal{M}_{s m n}^{(j)}(\mathbf{r})=\underline{\underline{B}} \cdot \mathcal{M}_{s m n}^{(j)}(\mathbf{r}), \\
& \mathbf{n}_{s m n}^{(j)}(\mathbf{r})=\underline{\underline{A}}^{-1} \cdot \mathcal{N}_{s m n}^{(j)}(\mathbf{r})=\underline{\underline{B}} \cdot \mathcal{N}_{s m n}^{(j)}(\mathbf{r}),
\end{aligned}
$$

with

$$
\underline{\underline{B}}=\alpha_{x} \hat{\mathbf{x}} \hat{\mathbf{x}}+\alpha_{y} \hat{\mathbf{y}} \hat{\mathbf{y}}+\hat{\mathbf{z}} \hat{\mathbf{z}}
$$


Using Eqs. (35)-(39), (50), and (51), we obtain

$$
\begin{aligned}
& \mathcal{M}_{s m n}^{(j)}(\mathbf{r})=\exp \left[i k_{0} \boldsymbol{\Gamma} \cdot \mathbf{r}\right] \frac{\mathcal{J}_{n}^{(j)}(k \mathbf{r})}{f_{1}(\phi)} \\
& \times\left\{\hat{\mathbf{r}}\left[\frac{f_{4}(\phi)-f_{1}^{2}(\phi)}{f_{2}(\theta, \phi)} \sin \theta \cos \theta \mathcal{Q}_{s m n}(\theta, \phi)-\left(\alpha_{x}-\alpha_{y}\right) \sin \theta \sin \phi \cos \phi \mathcal{R}_{s m n}(\theta, \phi)\right]\right. \\
& \quad+\hat{\boldsymbol{\theta}}\left[\frac{f_{4}(\phi) \cos ^{2} \theta+f_{1}^{2}(\phi) \sin ^{2} \theta}{f_{2}(\theta, \phi)} \mathcal{Q}_{s m n}(\theta, \phi)-\left(\alpha_{x}-\alpha_{y}\right) \cos \theta \sin \phi \cos \phi \mathcal{R}_{s m n}(\theta, \phi)\right] \\
& \left.\quad+\hat{\boldsymbol{\phi}}\left[-\frac{\alpha_{x}-\alpha_{y}}{f_{2}(\theta, \phi)} \cos \theta \sin \phi \cos \phi \mathcal{Q}_{s m n}(\theta, \phi)-f_{4}(\phi) \mathcal{R}_{s m n}(\theta, \phi)\right]\right\}
\end{aligned}
$$

and

$$
\begin{aligned}
\mathcal{N}_{s m n}^{(j)}(\mathbf{r})=\exp \left[i k_{0} \boldsymbol{\Gamma} \cdot \mathbf{r}\right] \\
\times\left(\hat { \mathbf { r } } \left\{\frac{\mathcal{J}_{n}^{(j)}(k \mathbf{r})}{k r}\left[\frac{\cos ^{2} \theta+f_{4}(\phi) \sin ^{2} \theta}{f_{2}^{2}(\theta, \phi)}\right] \mathcal{P}_{s m n}(\theta, \phi)\right.\right. \\
\left.+\quad \frac{\mathcal{K}_{n}^{(j)}(k \mathbf{r})}{f_{1}(\phi)}\left[\frac{f_{4}(\phi)-f_{1}^{2}(\phi)}{f_{2}(\theta, \phi)} \sin \theta \cos \theta \mathcal{R}_{s m n}(\theta, \phi)+\left(\alpha_{x}-\alpha_{y}\right) \sin \theta \sin \phi \cos \phi \mathcal{Q}_{s m n}(\theta, \phi)\right]\right\} \\
+\hat{\boldsymbol{\theta}}\left\{\frac{\mathcal{J}_{n}^{(j)}(k \mathbf{r})}{k r}\left[\frac{f_{4}(\phi)-1}{f_{2}^{2}(\theta, \phi)} \sin \theta \cos \theta\right] \mathcal{P}_{s m n}(\theta, \phi)\right. \\
\left.+\quad \frac{\mathcal{K}_{n}^{(j)}(k \mathbf{r})}{f_{1}(\phi)}\left[\frac{f_{4}(\phi) \cos ^{2} \theta+f_{1}^{2}(\phi) \sin ^{2} \theta}{f_{2}(\theta, \phi)} \mathcal{R}_{s m n}(\theta, \phi)+\left(\alpha_{x}-\alpha_{y}\right) \cos \theta \sin \phi \cos \phi \mathcal{Q}_{s m n}(\theta, \phi)\right]\right\} \\
+\hat{\boldsymbol{\phi}}\left\{-\frac{\mathcal{J}_{n}^{(j)}(k \mathbf{r})}{k r}\left[\frac{\alpha_{x}-\alpha_{y}}{f_{2}^{2}(\theta, \phi)} \sin \theta \sin \phi \cos \phi\right] \mathcal{P}_{s m n}(\theta, \phi)\right. \\
\left.\left.++\frac{\mathcal{K}_{n}^{(j)}(k \mathbf{r})}{f_{1}(\phi)}\left[-\frac{\alpha_{x}-\alpha_{y}}{f_{2}(\theta, \phi)} \cos \theta \sin \phi \cos \phi \mathcal{R}_{s m n}(\theta, \phi)+f_{4}(\phi) \mathcal{Q}_{s m n}(\theta, \phi)\right]\right\}\right) .
\end{aligned}
$$

Furthermore,

$$
\begin{aligned}
\underline{B}= & {\left[f_{4}(\phi) \sin ^{2} \theta+\cos ^{2} \theta\right] \hat{\mathbf{r}} \hat{\mathbf{r}}+\left[f_{4}(\phi) \cos ^{2} \theta+\sin ^{2} \theta\right] \hat{\boldsymbol{\theta}} \hat{\boldsymbol{\theta}}+\left[\alpha_{x}+\alpha_{y}-f_{4}(\phi)\right] \hat{\boldsymbol{\phi}} \hat{\boldsymbol{\phi}} } \\
& +\left[f_{4}(\phi)-1\right] \sin \theta \cos \theta(\hat{\mathbf{r}} \hat{\boldsymbol{\theta}}+\hat{\boldsymbol{\theta}} \hat{\mathbf{r}}) \\
& -\left(\alpha_{x}-\alpha_{y}\right) \sin \phi \cos \phi[\sin \theta(\hat{\mathbf{r}} \hat{\boldsymbol{\phi}}+\hat{\boldsymbol{\phi}} \hat{\mathbf{r}})+\cos \theta(\hat{\boldsymbol{\theta}} \hat{\boldsymbol{\phi}}+\hat{\boldsymbol{\phi}} \hat{\boldsymbol{\theta}})] .
\end{aligned}
$$

In the foregoing expressions, the following angular functions have been used:

$$
\begin{aligned}
& f_{1}(\phi)=+\left(\alpha_{x}^{2} \cos ^{2} \phi+\alpha_{y}^{2} \sin ^{2} \phi\right)^{1 / 2} \\
& f_{2}(\theta, \phi)=+\left[f_{1}^{2}(\phi) \sin ^{2} \theta+\cos ^{2} \theta\right]^{1 / 2} \\
& f_{3}(\phi)=\tan ^{-1}\left(\frac{\alpha_{y}}{\alpha_{x}} \tan \phi\right) \\
& f_{4}(\phi)=\alpha_{x} \cos ^{2} \phi+\alpha_{y} \sin ^{2} \phi .
\end{aligned}
$$


The angle $f_{3}(\phi)$ must lie in the same quadrant as its argument. Additionally,

$$
\begin{aligned}
& \mathcal{J}_{n}^{(1)}(k \mathbf{r})=j_{n}\left[k r f_{2}(\theta, \phi)\right], \\
& \mathcal{J}_{n}^{(3)}(k \mathbf{r})=h_{n}^{(1)}\left[k r f_{2}(\theta, \phi)\right], \\
& \mathcal{K}_{n}^{(j)}(k \mathbf{r})=\frac{n+1}{k r f_{2}(\theta, \phi)} \mathcal{J}_{n}^{(j)}(k \mathbf{r})-\mathcal{J}_{n+1}^{(j)}(k \mathbf{r}), \quad j \in\{1,3\}, \\
& \mathcal{P}_{s m n}(\theta, \phi)=n(n+1) P_{n}^{m}\left[\frac{\cos \theta}{f_{2}(\theta, \phi)}\right] \mathcal{V}_{s m}(\phi), \\
& \mathcal{Q}_{s m n}(\theta, \phi)=m P_{n}^{m}\left[\frac{\cos \theta}{f_{2}(\theta, \phi)}\right] \frac{f_{2}(\theta, \phi)}{f_{1}(\phi) \sin \theta} \mathcal{U}_{s m}(\phi), \\
& \mathcal{R}_{s m n}(\theta, \phi)=\frac{1}{f_{1}(\phi) \sin \theta}\left\{(n-m+1) f_{2}(\theta, \phi) P_{n+1}^{m}\left[\frac{\cos \theta}{f_{2}(\theta, \phi)}\right]\right. \\
& \mathcal{U}_{s m}(\phi)=\left\{\begin{array}{c}
-\sin \left[m f_{3}(\phi)\right] \\
\cos \left[m f_{3}(\phi)\right]
\end{array}\right\}, \quad s=\left\{\begin{array}{l}
e \\
\cos \left[m f_{3}(\phi)\right]
\end{array}\right\}, \quad s=\left\{\begin{array}{l}
e \\
o
\end{array} .\right. \\
& \mathcal{V}_{s m}(\phi)=\left\{\begin{array}{l}
\cos \theta P_{n}^{m}\left[m f_{3}(\phi)\right] \\
\sin [\theta, \phi)
\end{array}\right\} \mathcal{V}_{s m}(\phi),
\end{aligned}
$$

\subsubsection{Special case: $\alpha_{x}=\alpha_{y}=\alpha$}

When the diagonal dyadic $\underline{A}$ is simplified from biaxial $\left(\alpha_{x} \neq \alpha_{y}\right)$ to uniaxial $\left(\alpha_{x}=\alpha_{y}=\alpha\right)$, the functions $f_{1}$ to $f_{4}$ simplify considerably. The wavefunctions $\mathcal{M}_{s m n}^{(j)}(\mathbf{r})$ and $\mathcal{N}_{s m n}^{(j)}(\mathbf{r})$ then simplify as follows:

$$
\begin{gathered}
\mathcal{M}_{s m n}^{(j)}(\mathbf{r})=\exp \left[i k_{0} \boldsymbol{\Gamma} \cdot \mathbf{r}\right] \tilde{\mathcal{J}}_{n}^{(j)}(k \mathbf{r})\left\{-\hat{\mathbf{r}} \frac{\alpha-1}{f_{5}(\theta)} \sin \theta \cos \theta \tilde{\mathcal{Q}}_{s m n}(\theta, \phi)\right. \\
\left.+\hat{\boldsymbol{\theta}} \frac{\alpha \sin ^{2} \theta+\cos ^{2} \theta}{f_{5}(\theta)} \tilde{\mathcal{Q}}_{s m n}(\theta, \phi)-\hat{\boldsymbol{\phi}} \tilde{\mathcal{R}}_{s m n}(\theta, \phi)\right\}
\end{gathered}
$$

$$
\begin{aligned}
& \mathcal{N}_{s m n}^{(j)}(\mathbf{r})=\exp \left[i k_{0} \mathbf{\Gamma} \cdot \mathbf{r}\right] \\
& \quad \times\left\{\hat{\mathbf{r}}\left[\frac{\tilde{\mathcal{J}}_{n}^{(j)}(k \mathbf{r})}{k r} \frac{f_{6}(\theta)}{f_{5}^{2}(\theta)} \tilde{\mathcal{P}}_{s m n}(\theta, \phi)-\tilde{\mathcal{K}}_{n}^{(j)}(k \mathbf{r}) \frac{\alpha-1}{f_{5}(\theta)} \sin \theta \cos \theta \tilde{\mathcal{R}}_{s m n}(\theta, \phi)\right]\right. \\
& \quad+\hat{\boldsymbol{\theta}}\left[\frac{\tilde{\mathcal{J}}_{n}^{(j)}(k \mathbf{r})}{k r} \frac{\alpha-1}{f_{5}^{2}(\theta)} \sin \theta \cos \theta \tilde{\mathcal{P}}_{s m n}(\theta, \phi)+\tilde{\mathcal{K}}_{n}^{(j)}(k \mathbf{r}) \frac{f_{6}(\theta)}{f_{5}(\theta)} \tilde{\mathcal{R}}_{s m n}(\theta, \phi)\right] \\
& \left.\quad+\hat{\boldsymbol{\phi}} \tilde{\mathcal{K}}_{n}^{(j)}(k \mathbf{r}) \tilde{\mathcal{Q}}_{s m n}(\theta, \phi)\right\} .
\end{aligned}
$$

Similarly, $\underline{B}$ also simplifies to

$$
\underline{\underline{B}}=f_{6}(\theta) \hat{\mathbf{r}} \hat{\mathbf{r}}+\left[\alpha+1-f_{6}(\theta)\right] \hat{\boldsymbol{\theta}} \hat{\boldsymbol{\theta}}+\alpha \hat{\boldsymbol{\phi}} \hat{\boldsymbol{\phi}}+(\alpha-1) \sin \theta \cos \theta(\hat{\mathbf{r}} \hat{\boldsymbol{\theta}}+\hat{\boldsymbol{\theta}} \hat{\mathbf{r}})
$$


The following shorthand notation has been used:

$$
\begin{aligned}
& f_{5}(\theta)=+\left(\alpha^{2} \sin ^{2} \theta+\cos ^{2} \theta\right)^{1 / 2}, \\
& f_{6}(\theta)=\alpha \sin ^{2} \theta+\cos ^{2} \theta \\
& \tilde{\mathcal{J}}_{n}^{(1)}(k \mathbf{r})=j_{n}\left[k r f_{5}(\theta)\right] \\
& \tilde{\mathcal{J}}_{n}^{(3)}(k \mathbf{r})=h_{n}^{(1)}\left[k r f_{5}(\theta)\right] \\
& \tilde{\mathcal{K}}_{n}^{(j)}(k \mathbf{r})=\frac{n+1}{k r f_{5}(\theta)} \tilde{\mathcal{J}}_{n}^{(j)}(k \mathbf{r})-\tilde{\mathcal{J}}_{n+1}^{(j)}(k \mathbf{r}), \quad j \in\{1,3\}, \\
& \tilde{\mathcal{P}}_{s m n}(\theta, \phi)=n(n+1) P_{n}^{m}\left[\frac{\cos \theta}{f_{5}(\theta)}\right]\left\{\begin{array}{c}
\cos (m \phi) \\
\sin (m \phi)
\end{array}\right\}, \quad s=\left\{\begin{array}{l}
e \\
o
\end{array},\right. \\
& \tilde{\mathcal{Q}}_{s m n}(\theta, \phi)=m P_{n}^{m}\left[\frac{\cos \theta}{f_{5}(\theta)}\right] \frac{f_{5}(\theta)}{\alpha \sin \theta}\left\{\begin{array}{c}
-\sin (m \phi) \\
\cos (m \phi)
\end{array}\right\}, \quad s=\left\{\begin{array}{l}
e \\
o
\end{array},\right. \\
& \tilde{\mathcal{R}}_{s m n}(\theta, \phi)=\frac{1}{\alpha \sin \theta}\left\{(n-m+1) f_{5}(\theta) P_{n+1}^{m}\left[\frac{\cos \theta}{f_{5}(\theta)}\right]\right. \\
& \left.\quad-(n+1) \cos \theta P_{n}^{m}\left[\frac{\cos \theta}{f_{5}(\theta)}\right]\right\}\left\{\begin{array}{c}
\cos (m \phi) \\
\sin (m \phi)
\end{array}\right\}, \quad s=\left\{\begin{array}{l}
e \\
o
\end{array}\right.
\end{aligned}
$$

\subsubsection{Special case: $\alpha_{x}=\alpha_{y}=1$}

Further simplification to $\underline{\underline{A}}=\underline{\underline{I}}$ leads to $\underline{\underline{B}}=\underline{\underline{I}}$, so that

$$
\begin{aligned}
& \mathbf{m}_{s m n}^{(j)}(\mathbf{r})=\mathcal{M}_{s m n}^{(j)}(\mathbf{r})=\exp \left(i k_{0} \boldsymbol{\Gamma} \cdot \mathbf{r}\right) \mathbf{M}_{s m n}^{(j)}(k \mathbf{r}), \\
& \mathbf{n}_{s m n}^{(j)}(\mathbf{r})=\mathcal{N}_{s m n}^{(j)}(\mathbf{r})=\exp \left(i k_{0} \boldsymbol{\Gamma} \cdot \mathbf{r}\right) \mathbf{N}_{s m n}^{(j)}(k \mathbf{r}) .
\end{aligned}
$$

\subsection{T matrix for scattering by a $3 \mathrm{D}$ anisotropic object}

For an example of the use of the derived vector spherical wavefunctions, let us now consider scattering by a $3 \mathrm{D}$ object that occupies the region $\mathrm{V}$ and has a surface $\mathrm{S}$. The material occupying $\mathrm{V}$ obeys the constitutive relations (6) and (7). The coordinate system is chosen so that its origin lies inside $\mathrm{V}$ and the conditions (54) hold. The external region is vacuous.

Suppose that an electromagnetic field is incident on the scatterer. This incident field is purely arbitrary except that its source must lie outside a minimum sphere centered at the origin and circumscribing V. It can then be represented as [22]

$$
\begin{aligned}
& \mathbf{E}_{i n c}(\mathbf{r})=\sum_{s=e, o} \sum_{n=1}^{\infty} \sum_{m=0}^{n} D_{m n}\left[A_{s m n}^{(1)} \mathbf{M}_{s m n}^{(1)}\left(k_{0} \mathbf{r}\right)+B_{s m n}^{(1)} \mathbf{N}_{s m n}^{(1)}\left(k_{0} \mathbf{r}\right)\right], \\
& \mathbf{H}_{i n c}(\mathbf{r})=-\frac{i}{\eta_{0}} \sum_{s=e, o} \sum_{n=1}^{\infty} \sum_{m=0}^{n} D_{m n}\left[A_{s m n}^{(1)} \mathbf{N}_{s m n}^{(1)}\left(k_{0} \mathbf{r}\right)+B_{s m n}^{(1)} \mathbf{M}_{s m n}^{(1)}\left(k_{0} \mathbf{r}\right)\right],
\end{aligned}
$$

where the coefficients $A_{s m n}^{(1)}$ and $B_{s m n}^{(1)}$ are presumed to be known and the normalization constant

$$
D_{m n}=\left(2-\delta_{m 0}\right) \frac{2 n+1}{4 n(n+1)} \frac{(n-m) !}{(n+m) !},
$$

involves the Kronecker delta $\delta_{p q}$. Outside the circumscribing sphere, the scattered electromagnetic field 
is represented by the sums

$$
\begin{aligned}
& \mathbf{E}_{s c a}(\mathbf{r})=\sum_{s=e, o} \sum_{n=1}^{\infty} \sum_{m=0}^{n} D_{m n}\left[A_{s m n}^{(3)} \mathbf{M}_{s m n}^{(3)}\left(k_{0} \mathbf{r}\right)+B_{s m n}^{(3)} \mathbf{N}_{s m n}^{(3)}\left(k_{0} \mathbf{r}\right)\right], \\
& \mathbf{H}_{s c a}(\mathbf{r})=-\frac{i}{\eta_{0}} \sum_{s=e, o} \sum_{n=1}^{\infty} \sum_{m=0}^{n} D_{m n}\left[A_{s m n}^{(3)} \mathbf{N}_{s m n}^{(3)}\left(k_{0} \mathbf{r}\right)+B_{s m n}^{(3)} \mathbf{M}_{s m n}^{(3)}\left(k_{0} \mathbf{r}\right)\right],
\end{aligned}
$$

where the unknown coefficients $A_{s m n}^{(3)}$ and $B_{s m n}^{(3)}$ have to be determined. The underlying presumption here is that the source of the incident field lies so far away from the scattering object that any action of the scattered field on that source is vanishingly small.

Application of the Ewald-Oseen extinction theorem followed by some analytic-continuation arguments leads to the following four relationships $[22,23]$ :

$$
\begin{aligned}
& A_{s m n}^{(1)}=-\frac{i k_{0}^{2}}{\pi} \int_{\mathbf{S}} d^{2} \mathbf{r}_{p}\left\{\left[\mathbf{n}\left(\mathbf{r}_{p}\right) \times \mathbf{E}_{i n t}\left(\mathbf{r}_{p}\right)\right] \cdot \mathbf{N}_{s m n}^{(3)}\left(k_{0} \mathbf{r}_{p}\right)\right. \\
& \left.+i \eta_{0}\left[\mathbf{n}\left(\mathbf{r}_{p}\right) \times \mathbf{H}_{i n t}\left(\mathbf{r}_{p}\right)\right] \cdot \mathbf{M}_{s m n}^{(3)}\left(k_{0} \mathbf{r}_{p}\right)\right\} \\
& B_{s m n}^{(1)}=-\frac{i k_{0}^{2}}{\pi} \int_{\mathbf{S}} d^{2} \mathbf{r}_{p}\left\{\left[\mathbf{n}\left(\mathbf{r}_{p}\right) \times \mathbf{E}_{i n t}\left(\mathbf{r}_{p}\right)\right] \cdot \mathbf{M}_{s m n}^{(3)}\left(k_{0} \mathbf{r}_{p}\right)\right. \\
& \left.+i \eta_{0}\left[\mathbf{n}\left(\mathbf{r}_{p}\right) \times \mathbf{H}_{i n t}\left(\mathbf{r}_{p}\right)\right] \cdot \mathbf{N}_{s m n}^{(3)}\left(k_{0} \mathbf{r}_{p}\right)\right\} \\
& A_{s m n}^{(3)}=\frac{i k_{0}^{2}}{\pi} \int_{\mathbf{S}} d^{2} \mathbf{r}_{p}\left\{\left[\mathbf{n}\left(\mathbf{r}_{p}\right) \times \mathbf{E}_{i n t}\left(\mathbf{r}_{p}\right)\right] \cdot \mathbf{N}_{s m n}^{(1)}\left(k_{0} \mathbf{r}_{p}\right)\right. \\
& \left.+i \eta_{0}\left[\mathbf{n}\left(\mathbf{r}_{p}\right) \times \mathbf{H}_{i n t}\left(\mathbf{r}_{p}\right)\right] \cdot \mathbf{M}_{s m n}^{(1)}\left(k_{0} \mathbf{r}_{p}\right)\right\} \\
& B_{s m n}^{(3)}=\frac{i k_{0}^{2}}{\pi} \int_{\mathbf{S}} d^{2} \mathbf{r}_{p}\left\{\left[\mathbf{n}\left(\mathbf{r}_{p}\right) \times \mathbf{E}_{i n t}\left(\mathbf{r}_{p}\right)\right] \cdot \mathbf{M}_{s m n}^{(1)}\left(k_{0} \mathbf{r}_{p}\right)\right. \\
& \left.+i \eta_{0}\left[\mathbf{n}\left(\mathbf{r}_{p}\right) \times \mathbf{H}_{i n t}\left(\mathbf{r}_{p}\right)\right] \cdot \mathbf{N}_{s m n}^{(1)}\left(k_{0} \mathbf{r}_{p}\right)\right\}
\end{aligned}
$$

Here, $\mathbf{n}\left(\mathbf{r}_{p}\right)$ is the unit outward normal to $\mathbf{S}$ at $\mathbf{r}_{p} \in \mathbf{S}$, whereas $\mathbf{E}_{\text {int }}(\mathbf{r})$ and $\mathbf{H}_{\text {int }}(\mathbf{r})$ are the fields excited inside the scatterer.

A representation of the internal fields emerges from Eqs. (52), (53), (55), and (56) as

$$
\begin{aligned}
& \mathbf{E}_{i n t}(\mathbf{r})=\underline{\underline{B}} \cdot \sum_{s=e, o} \sum_{n=1}^{\infty} \sum_{m=0}^{n}\left[b_{s m n}^{(1)} \mathcal{M}_{s m n}^{(1)}(\mathbf{r})+c_{s m n}^{(1)} \mathcal{N}_{s m n}^{(1)}(\mathbf{r})\right], \\
& \mathbf{H}_{i n t}(\mathbf{r})=-\frac{i}{\eta_{0} \eta_{r}} \underline{\underline{B}} \cdot \sum_{s=e, o} \sum_{n=1}^{\infty} \sum_{m=0}^{n}\left[c_{s m n}^{(1)} \mathcal{M}_{s m n}^{(1)}(\mathbf{r})+b_{s m n}^{(1)} \mathcal{N}_{s m n}^{(1)}(\mathbf{r})\right],
\end{aligned}
$$

because the internal fields must be regular at the origin $\mathbf{0} \in \mathrm{V}$.

Substitution of Eqs. (95) and (96) in Eqs. (91) and (92) leads to the matrix equation written compactly as

$$
\begin{aligned}
{\left[\begin{array}{c}
A_{s m n}^{(1)} \\
---- \\
B_{s m n}^{(1)}
\end{array}\right] } & =\left[\begin{array}{c|c}
I_{s m n, s^{\prime} m^{\prime} n^{\prime}}^{(1)} & J_{s m n, s^{\prime} m^{\prime} n^{\prime}}^{(1)} \\
------ & ------ \\
K_{s m n, s^{\prime} m^{\prime} n^{\prime}}^{(1)} & L_{s m n, s^{\prime} m^{\prime} n^{\prime}}^{(1)}
\end{array}\right]\left[\begin{array}{c}
b_{s^{\prime} m^{\prime} n^{\prime}}^{(1)} \\
---- \\
c_{s^{\prime} m^{\prime} n^{\prime}}^{(1)}
\end{array}\right] \\
& \equiv\left[Y^{(1)}\right]\left[\begin{array}{c}
b_{s^{\prime} m^{\prime} n^{\prime}}^{(1)} \\
---- \\
c_{s^{\prime} m^{\prime} n^{\prime}}^{(1)}
\end{array}\right]
\end{aligned}
$$


likewise, Eqs. (93)-(96) yield written compactly as

$$
\begin{aligned}
{\left[\begin{array}{c}
A_{s m n}^{(3)} \\
---- \\
B_{s m n}^{(3)}
\end{array}\right] } & =-\left[\begin{array}{c|c}
I_{s m n, s^{\prime} m^{\prime} n^{\prime}}^{(3)} & J_{s m n, s^{\prime} m^{\prime} n^{\prime}}^{(3)} \\
------ & ------ \\
K_{s m n, s^{\prime} m^{\prime} n^{\prime}}^{(3)} & L_{s m n, s^{\prime} m^{\prime} n^{\prime}}^{(3)}
\end{array}\right]\left[\begin{array}{c}
b_{s^{\prime} m^{\prime} n^{\prime}}^{(1)} \\
---- \\
c_{s^{\prime} m^{\prime} n^{\prime}}^{(1)}
\end{array}\right] \\
& \equiv-\left[Y^{(3)}\right]\left[\begin{array}{c}
b_{s^{\prime} m^{\prime} n^{\prime}}^{(1)} \\
---- \\
c_{s^{\prime} m^{\prime} n^{\prime}}^{(1)}
\end{array}\right] .
\end{aligned}
$$

These relations require the computation of the integrals

$$
\begin{aligned}
& I_{s m n, s^{\prime} m^{\prime} n^{\prime}}^{(j)}=-\frac{i k_{0}^{2}}{\pi} \int_{\mathbf{S}} d^{2} \mathbf{r}_{p}\left(\mathbf{N}_{s m n}^{(\ell)}\left(k_{0} \mathbf{r}_{p}\right) \cdot\left\{\mathbf{n}\left(\mathbf{r}_{p}\right) \times\left[\underline{\underline{B}} \cdot \mathcal{M}_{s^{\prime} m^{\prime} n^{\prime}}^{(1)}\left(\mathbf{r}_{p}\right)\right]\right\}\right. \\
& \left.\quad+\frac{1}{\eta_{r}} \mathbf{M}_{s m n}^{(\ell)}\left(k_{0} \mathbf{r}_{p}\right) \cdot\left\{\mathbf{n}\left(\mathbf{r}_{p}\right) \times\left[\underline{\underline{B}} \cdot \mathcal{N}_{s^{\prime} m^{\prime} n^{\prime}}^{(1)}\left(\mathbf{r}_{p}\right)\right]\right\}\right), \\
& J_{s m n, s^{\prime} m^{\prime} n^{\prime}}^{(j)}=-\frac{i k_{0}^{2}}{\pi} \int_{\mathrm{S}} d^{2} \mathbf{r}_{p}\left(\mathbf{N}_{s m n}^{(\ell)}\left(k_{0} \mathbf{r}_{p}\right) \cdot\left\{\mathbf{n}\left(\mathbf{r}_{p}\right) \times\left[\underline{\underline{B}} \cdot \mathcal{N}_{s^{\prime} m^{\prime} n^{\prime}}^{(1)}\left(\mathbf{r}_{p}\right)\right]\right\}\right. \\
& \left.\quad+\frac{1}{\eta_{r}} \mathbf{M}_{s m n}^{(\ell)}\left(k_{0} \mathbf{r}_{p}\right) \cdot\left\{\mathbf{n}\left(\mathbf{r}_{p}\right) \times\left[\underline{\underline{B}} \cdot \mathcal{M}_{s^{\prime} m^{\prime} n^{\prime}}^{(1)}\left(\mathbf{r}_{p}\right)\right]\right\}\right), \\
& K_{s m n, s^{\prime} m^{\prime} n^{\prime}}^{(j)}=-\frac{i k_{0}^{2}}{\pi} \int_{\mathrm{S}} d^{2} \mathbf{r}_{p}\left(\mathbf{M}_{s m n}^{(\ell)}\left(k_{0} \mathbf{r}_{p}\right) \cdot\left\{\mathbf{n}\left(\mathbf{r}_{p}\right) \times\left[\underline{\underline{B}} \cdot \mathcal{M}_{s^{\prime} m^{\prime} n^{\prime}}^{(1)}\left(\mathbf{r}_{p}\right)\right]\right\}\right. \\
& \left.+\frac{1}{\eta_{r}} \mathbf{N}_{s m n}^{(\ell)}\left(k_{0} \mathbf{r}_{p}\right) \cdot\left\{\mathbf{n}\left(\mathbf{r}_{p}\right) \times\left[\underline{\underline{B}} \cdot \mathcal{N}_{s^{\prime} m^{\prime} n^{\prime}}^{(1)}\left(\mathbf{r}_{p}\right)\right]\right\}\right), \\
& L_{s m n, s^{\prime} m^{\prime} n^{\prime}}^{(j)}=-\frac{i k_{0}^{2}}{\pi} \int_{\mathrm{S}} d^{2} \mathbf{r}_{p}\left(\mathbf{M}_{s m n}^{(\ell)}\left(k_{0} \mathbf{r}_{p}\right) \cdot\left\{\mathbf{n}\left(\mathbf{r}_{p}\right) \times\left[\underline{\underline{B}} \cdot \mathcal{N}_{s^{\prime} m^{\prime} n^{\prime}}^{(1)}\left(\mathbf{r}_{p}\right)\right]\right\}\right. \\
& \left.+\frac{1}{\eta_{r}} \mathbf{N}_{s m n}^{(\ell)}\left(k_{0} \mathbf{r}_{p}\right) \cdot\left\{\mathbf{n}\left(\mathbf{r}_{p}\right) \times\left[\underline{\underline{B}} \cdot \mathcal{M}_{s^{\prime} m^{\prime} n^{\prime}}^{(1)}\left(\mathbf{r}_{p}\right)\right]\right\}\right),
\end{aligned}
$$

where $j \in\{1,3\}$ and $\ell=j+2(\bmod 4) \in\{3,1\}$.

Equations (97) and (98) yield a matrix relationship between the incident-field coefficients and the scattered-field coefficients that can be expressed as

$$
\left[\begin{array}{c}
A_{s m n}^{(3)} \\
---- \\
B_{s m n}^{(3)}
\end{array}\right]=[T]\left[\begin{array}{c}
A_{s m n}^{(1)} \\
---- \\
B_{s m n}^{(1)}
\end{array}\right],
$$

where the T matrix

$$
[T]=-\left[Y^{(3)}\right]\left[Y^{(1)}\right]^{-1}
$$

completely characterizes the scattering response of the 3D anisotropic object.

The calculation of the T matrix of a 3D object made of a material obeying the constitutive relations (6) and (7) along with (54) is a major exercise that we intend to take up shortly. Let us, however, mention that our derivation procedure can be extended to cover the possibility that the external region is occupied by a homogeneous isotropic material which can be dielectric-magnetic, chiral, or even biisotropic [24]. 


\section{Radiation fields}

Returning to Eqs. (8) and (9), we are now interested in finding the electric field radiated by a source current density distribution. Accordingly,

$$
\mathbf{E}(\mathbf{r})=i \omega \mu_{0} \mu_{r} \iiint d^{3} \mathbf{r}_{p} \underline{\underline{G}}\left(\mathbf{r}, \mathbf{r}_{p}\right) \cdot \mathbf{J}\left(\mathbf{r}_{p}\right),
$$

where $\underline{\underline{G}}\left(\mathbf{r}, \mathbf{r}_{p}\right)$ is the dyadic Green function with $\mathbf{r}$ as the field point and $\mathbf{r}_{p}$ as the source point.

\subsection{Dyadic Green function: Coordinate-free form}

Our starting point is the particular solution of Eq. (28), which is known to be [25]

$$
\tilde{\mathbf{e}}\left(\mathbf{r}^{\prime}\right)=i \omega \mu_{0} \mu_{r} A \iiint d^{3} \mathbf{r}_{p}^{\prime} \underline{\underline{g}}\left(\mathbf{r}^{\prime}, \mathbf{r}_{p}^{\prime}\right) \cdot \tilde{\mathbf{j}}\left(\mathbf{r}_{p}^{\prime}\right)
$$

where

$$
\underline{\underline{g}}\left(\mathbf{r}^{\prime}, \mathbf{r}_{p}^{\prime}\right)=\left(\underline{\underline{I}}+k^{-2} \nabla^{\prime} \nabla^{\prime}\right) \frac{\exp \left(i k\left|\mathbf{r}^{\prime}-\mathbf{r}_{p}^{\prime}\right|\right)}{4 \pi\left|\mathbf{r}^{\prime}-\mathbf{r}_{p}^{\prime}\right|}
$$

Inverting the relations (23) and (25), we get

$$
\mathbf{e}^{\prime}\left(\mathbf{r}^{\prime}\right)=i \omega \mu_{0} \mu_{r} \iiint d^{3} \mathbf{r}_{p}^{\prime} \underline{\underline{g^{\prime}}}\left(\mathbf{r}^{\prime}, \mathbf{r}_{p}^{\prime}\right) \cdot \mathbf{j}^{\prime}\left(\mathbf{r}_{p}^{\prime}\right)
$$

from Eq. (106), where

$$
\begin{aligned}
\underline{\underline{g}}\left(\mathbf{r}^{\prime}, \mathbf{r}_{p}^{\prime}\right) & =A^{3} \underline{\underline{A}}^{-1} \cdot \underline{\underline{g}}\left(\underline{\underline{A}}^{-1} \cdot \mathbf{r}^{\prime}, \underline{\underline{A}}^{-1} \cdot \mathbf{r}_{p}^{\prime}\right) \cdot \underline{\underline{A}}^{-1} \\
& =A^{3} \underline{\underline{A}}^{-1} \cdot\left(\underline{\underline{I}}+k^{-2} \underline{\underline{A}} \cdot \nabla^{\prime} \nabla^{\prime} \cdot \underline{\underline{A}}\right) \cdot \underline{\underline{A}}^{-1} \frac{\exp \left[i k\left|\underline{\underline{A}}^{-1} \cdot\left(\mathbf{r}^{\prime}-\mathbf{r}_{p}^{\prime}\right)\right|\right]}{4 \pi\left|\underline{\underline{A^{-1}}} \cdot\left(\mathbf{r}^{\prime}-\mathbf{r}_{p}^{\prime}\right)\right|} \\
& =A^{3}\left(\underline{\underline{A}}^{-2}+k^{-2} \nabla^{\prime} \nabla^{\prime}\right) \frac{\exp \left[i k\left|\underline{\underline{A}}^{-1} \cdot\left(\mathbf{r}^{\prime}-\mathbf{r}_{p}^{\prime}\right)\right|\right]}{4 \pi\left|\underline{\underline{A}}^{-1} \cdot\left(\mathbf{r}^{\prime}-\mathbf{r}_{p}^{\prime}\right)\right|}
\end{aligned}
$$

and the identity $\underline{\underline{A}}^{T}=\underline{\underline{A}}$ has been used.

Next, an inversion involving Eqs. (15) and (17) transforms Eq. (108) to

$$
\mathbf{e}(\mathbf{r})=i \omega \mu_{0} \mu_{r} \iiint d^{3} \mathbf{r}_{p} \underline{\underline{g}}\left(\mathbf{r}, \mathbf{r}_{p}\right) \cdot \mathbf{j}\left(\mathbf{r}_{p}\right)
$$

where

$$
\begin{aligned}
& \underline{\underline{g}}\left(\mathbf{r}, \mathbf{r}_{p}\right)=\underline{\underline{S}} \cdot \underline{\underline{g^{\prime}}}\left(\underline{\underline{S}} \cdot \mathbf{r}, \underline{\underline{S}} \cdot \mathbf{r}_{p}\right) \cdot \underline{\underline{S}}^{-1} \\
& =A^{3} \underline{\underline{S}} \cdot\left(\underline{\underline{A}}^{-2}+k^{-2} \underline{\underline{S}} \cdot \nabla \nabla \cdot \underline{\underline{S}}^{-1}\right) \cdot \underline{\underline{S}}^{-1} \frac{\exp \left[i k\left|\underline{\underline{A}}^{-1} \cdot \underline{\underline{S}} \cdot\left(\mathbf{r}-\mathbf{r}_{p}\right)\right|\right]}{4 \pi\left|\underline{\underline{A}}^{-1} \cdot \underline{\underline{S}} \cdot\left(\mathbf{r}-\mathbf{r}_{p}\right)\right|} \\
& =A^{3}\left(\underline{\underline{S}} \cdot \underline{\underline{A}}^{-2} \cdot \underline{\underline{S}}^{-1}+k^{-2} \underline{\underline{S}}^{2} \cdot \nabla \nabla \cdot \underline{\underline{S}}^{-2}\right) \frac{\exp \left[i k\left|\underline{\underline{A}}^{-1} \cdot \underline{\underline{S}} \cdot\left(\mathbf{r}-\mathbf{r}_{p}\right)\right|\right]}{4 \pi\left|\underline{\underline{A}}^{-1} \cdot \underline{\underline{S}} \cdot\left(\mathbf{r}-\mathbf{r}_{p}\right)\right|} .
\end{aligned}
$$

Finally, the use of Eqs. (10) and (12) in Eq. (112) yields Eq. (105) with

$$
\begin{aligned}
\underline{\underline{G}}\left(\mathbf{r}, \mathbf{r}_{p}\right)= & \exp \left[i k_{0} \boldsymbol{\Gamma} \cdot\left(\mathbf{r}-\mathbf{r}_{p}\right)\right] \underline{\underline{g}}\left(\mathbf{r}, \mathbf{r}_{p}\right) \\
= & A^{3} \exp \left[i k_{0} \boldsymbol{\Gamma} \cdot\left(\mathbf{r}-\mathbf{r}_{p}\right)\right]\left(\underline{\underline{S}} \cdot \underline{\underline{A}}^{-2} \cdot \underline{\underline{S}}^{-1}\right. \\
& \left.\quad+k^{-2} \underline{\underline{S}}^{2} \cdot \nabla \nabla \cdot \underline{\underline{S}}^{-2}\right) \frac{\exp \left[i k\left|\underline{\underline{A}}^{-1} \cdot \underline{\underline{S}} \cdot\left(\mathbf{r}-\mathbf{r}_{p}\right)\right|\right]}{4 \pi\left|\underline{\underline{A}}^{-1} \cdot \underline{\underline{S}} \cdot\left(\mathbf{r}-\mathbf{r}_{p}\right)\right|}
\end{aligned}
$$


in a coordinate-free form.

\subsection{Dyadic Green function: Singularity}

When the source point $\mathbf{r}_{p}$ and the field point $\mathbf{r}$ coincide, the dyadic Green function exhibits a singularity. In order to estimate this singularity, the source point is made the centroid of an electrically small convex region $\mathrm{V}_{\ell}$ bounded by the surface $\mathrm{S}_{\ell}$, which is then shrunk in volume isotropically [26]. This process is used to determine the depolarization dyadic $\underline{\underline{D}}$ defined from $\underline{\underline{G}}\left(\mathbf{r}, \mathbf{r}_{p}\right)$ via

$$
\lim _{\ell \rightarrow 0} \iiint_{\mathrm{V}_{\ell}} d^{3} \mathbf{r}_{q} \underline{\underline{G}}\left(\mathbf{r}_{p}, \mathbf{r}_{q}\right) \cdot \mathbf{b}\left(\mathbf{r}_{q}\right) \simeq \underline{\underline{D}} \cdot \mathbf{b}\left(\mathbf{r}_{p}\right)
$$

where the surface

$$
\mathrm{S}_{\ell}=\left\{\mathbf{r}_{q} \mid \mathbf{r}_{q}\left(\Omega_{\ell}\right)=\mathbf{r}_{p}+\ell \underline{\underline{U}} \ell \cdot \mathbf{u}_{\ell}\left(\Omega_{\ell}\right), \Omega_{\ell} \in[0,4 \pi]\right\} .
$$

Here, the positive scalar $\ell$ is a linear measure of the volume of $\mathrm{V}_{\ell}$; the dyadic $\underline{U}$ delineating the shape of $\mathrm{V}_{\ell}$ has a determinant equal to unity, while $\mathbf{u}_{\ell}$ and $\Omega_{\ell}$ denote, respectively, the radial unit vector and the solid angle in a spherical coordinate system with its origin at $\mathbf{r}_{p} \in \mathrm{V}_{\ell}$. The depolarization dyadic $\underline{\underline{D}}$ depends on the choice of $\underline{\underline{U}}_{\ell}[26]$.

In the present instance, regardless of the choice of $\underline{\underline{U}}_{\ell}$, two different approaches [27] to determining $\underline{\underline{D}}$ are possible as follows:

(a) Determine the analogous depolarization dyadic $\underline{\underline{d}}$ arising from $\underline{\tilde{g}}\left(\mathbf{r}^{\prime}, \mathbf{r}_{p}^{\prime}\right)$, and then reverse the three transformations described in Secs. 2.1-2.3 to obtain $\underline{\underline{D}}$. Implementing this indirect approach, we find that Eq. (107) leads to

$$
\underline{\underline{\underline{d}}}=-k^{-2} \underline{\underline{\underline{L}}}
$$

where

$$
\underline{\underline{L}}=\iint_{\mathrm{S}_{\ell}} d^{2} \mathbf{r}_{q} \frac{\mathbf{u}_{q} \mathbf{r}_{q}-\mathbf{u}_{q} \mathbf{r}_{p}}{4 \pi\left|\mathbf{r}_{q}-\mathbf{r}_{p}\right|^{3}}
$$

and $\mathbf{u}_{q}$ is the unit outward normal to $\mathrm{S}_{\ell}$ at $\mathbf{r}_{q} \in \mathrm{S}_{\ell}$. The dyadic $\underline{\underline{L}}$ is symmetric [26]. If $\mathrm{V}_{\ell}$ is either spherical or cubical, $\underline{\underline{L}}=(1 / 3) \underline{\underline{I}}$. For ellipsoidal $\mathrm{V}_{\ell}$, the dyadic $\underline{\underline{\underline{L}}}$ can be cast in terms of elliptic integrals that have to be evaluated numerically [28,29]. Using Eqs. (109) and (120), we get the

$$
\underline{\underline{d}}^{\prime}=-k^{-2} A^{3} \underline{\underline{A}}^{-1} \cdot \underline{\underline{L}} \cdot \underline{\underline{A}}^{-1}
$$

arising from $\underline{\underline{g^{\prime}}}\left(\mathbf{r}^{\prime}, \mathbf{r}_{p}^{\prime}\right)$, and finally

$$
\underline{\underline{D}}=-k^{-2} A^{3} \underline{\underline{S}} \cdot \underline{\underline{A}}^{-1} \cdot \underline{\underline{L}} \cdot \underline{\underline{A}}^{-1} \cdot \underline{\underline{S}}^{-1}
$$

emerges from the use of Eqs. (113) and (116).

(b) Directly obtain $\underline{D}$ for the material described by the constitutive relations (6) and (7), using the method of Michel and Weiglhofer [30]. This direct approach was formulated for ellipsoidal $\mathrm{V}_{\ell}$ and yields $[30]$

$$
\underline{\underline{D}}=-\frac{A^{2}}{4 \pi k^{2}} \int_{\varphi_{\rho}=0}^{2 \pi} d \varphi_{\rho} \int_{\vartheta_{\rho}=0}^{\pi} d \vartheta_{\rho} \sin \vartheta_{\rho} \frac{\underline{\underline{U_{l}}}{ }_{\ell}^{-1} \cdot \hat{\boldsymbol{\rho}} \hat{\boldsymbol{\rho}} \cdot \underline{\underline{U}}_{\ell}^{-1}}{\underline{\underline{U_{\ell}^{-1}}} \cdot \underline{\underline{S}} \cdot \underline{\underline{A}} \cdot \underline{\underline{A}} \cdot \underline{\underline{S}}^{-1} \cdot \underline{\underline{U}}_{\ell}^{-1} \cdot \hat{\boldsymbol{\rho}}},
$$

where $\hat{\boldsymbol{\rho}}=\left(\hat{\mathbf{x}} \cos \varphi_{\rho}+\hat{\mathbf{y}} \sin \varphi_{\rho}\right) \sin \vartheta_{\rho}+\hat{\mathbf{z}} \sin \vartheta_{\rho}$. 
For spherical $\vee_{\ell}$, the right side of Eq. (123) simplifies to $-\left(A^{3} / 3 k^{2}\right) \underline{\underline{S}} \cdot \underline{\underline{A}} \underline{\underline{ }}^{2} \cdot \underline{S}$, but the integral on the right side Eq. (124) has still to be expressed in terms of elliptic integrals [31]. Therefore, the depolarization dyadics (123) and (124) - obtained by two different approaches - are clearly distinct in general. The difference arises because, for the two approaches,

(i) $V_{\ell}$ was chosen in different spaces: $\mathbf{r}^{\prime}$ in the indirect approach and $\mathbf{r}$ in the direct approach; and

(ii) the singularity was considered for different fields $\tilde{\mathbf{e}}$ in the indirect approach and $\mathbf{e}$ (or $\mathbf{E}$ ) in the direct approach.

Knowledge of the depolarization dyadic will be useful in setting up the Maxwell Garnett and the Bruggeman formalisms to predict the effective constitutive parameters of a particulate composite material containing electrically small particles made of the chosen material [32].

\subsection{Dyadic Green function: Bilinear expansion}

Computation of the field radiated by a source can be greatly convenienced by the formulation of a blinear expression of the dyadic Green function $\underline{\underline{G}}\left(\mathbf{r}, \mathbf{r}_{p}\right)$, because the bilinear expression is a sum over the products of the derived vector spherical wavefunctions of the source point and the field point [33,34]. It is applicable only if the source point and the field point are situated at different distances from the origin.

We begin with the known bilinear expansion $[22,23]$

$$
\underline{\underline{g}}\left(\mathbf{r}^{\prime}, \mathbf{r}_{p}^{\prime}\right)=\frac{i k}{\pi} \sum_{s=e, o} \sum_{n=1}^{\infty} \sum_{m=0}^{n} D_{m n}\left[\mathbf{M}_{s m n}^{(3)}\left(k \mathbf{r}_{>}^{\prime}\right) \mathbf{M}_{s m n}^{(1)}\left(k \mathbf{r}_{<}^{\prime}\right)+\mathbf{N}_{s m n}^{(3)}\left(k \mathbf{r}_{>}^{\prime}\right) \mathbf{N}_{s m n}^{(1)}\left(k \mathbf{r}_{<}^{\prime}\right)\right],
$$

where

$$
\mathbf{r}_{>}^{\prime}=\left\{\begin{array}{ll}
\mathbf{r}^{\prime}, & r^{\prime}>r_{p}^{\prime} \\
\mathbf{r}_{p}^{\prime}, & r^{\prime}<r_{p}^{\prime}
\end{array}, \quad \mathbf{r}_{<}^{\prime}=\left\{\begin{array}{ll}
\mathbf{r}_{p}^{\prime}, & r^{\prime}>r_{p}^{\prime} \\
\mathbf{r}^{\prime}, & r^{\prime}<r_{p}^{\prime}
\end{array} .\right.\right.
$$

By following the process described in Sec. 4.1, we get

$$
\begin{aligned}
\underline{\underline{g}}\left(\mathbf{r}^{\prime}, \mathbf{r}_{p}^{\prime}\right) & =\frac{i k A^{3}}{\pi} \underline{\underline{A}}^{-1} \cdot \sum_{s=e, o} \sum_{n=1}^{\infty} \sum_{m=0}^{n} D_{m n}\left[\mathbf{M}_{s m n}^{(3)}\left(k \underline{\underline{A}}^{-1} \cdot \mathbf{r}_{>}^{\prime}\right) \mathbf{M}_{s m n}^{(1)}\left(k \underline{\underline{A}}^{-1} \cdot \mathbf{r}_{<}^{\prime}\right)\right. \\
& \left.+\mathbf{N}_{s m n}^{(3)}\left(k \underline{\underline{A}}^{-1} \cdot \mathbf{r}_{>}^{\prime}\right) \mathbf{N}_{s m n}^{(1)}\left(k \underline{\underline{A}}^{-1} \cdot \mathbf{r}_{<}^{\prime}\right)\right] \cdot \underline{\underline{A}}^{-1},
\end{aligned}
$$

and

$$
\begin{aligned}
\underline{\underline{g}}\left(\mathbf{r}, \mathbf{r}_{p}\right)= & \frac{i k A^{3}}{\pi} \exp \left[-i k_{0} \boldsymbol{\Gamma} \cdot\left(\mathbf{r}_{>}+\mathbf{r}_{<}\right)\right] \\
& \times \sum_{s=e, o} \sum_{n=1}^{\infty} \sum_{m=0}^{n} D_{m n}\left[\mathbf{m}_{s m n}^{(3)}\left(\mathbf{r}_{>}\right) \mathbf{m}_{s m n}^{(1)}\left(\mathbf{r}_{<}\right)+\mathbf{n}_{s m n}^{(3)}\left(\mathbf{r}_{>}\right) \mathbf{n}_{s m n}^{(1)}\left(\mathbf{r}_{<}\right)\right]
\end{aligned}
$$

where

$$
\mathbf{r}_{>}=\left\{\begin{array}{ll}
\mathbf{r}, & r>r_{p} \\
\mathbf{r}_{p}, & r<r_{p}
\end{array}, \quad \mathbf{r}_{<}=\left\{\begin{array}{ll}
\mathbf{r}_{p}, & r>r_{p} \\
\mathbf{r}, & r<r_{p}
\end{array} .\right.\right.
$$

Finally, from Eqs. (116) and (128), we get the desired bilinear expansion

$$
\begin{aligned}
& \underline{\underline{G}}\left(\mathbf{r}, \mathbf{r}_{p}\right)=\frac{i k A^{3}}{\pi} \exp \left[-i k_{0} \boldsymbol{\Gamma} \cdot\left(\mathbf{r}_{>}+\mathbf{r}_{<}-\mathbf{r}+\mathbf{r}_{p}\right)\right] \\
& \quad \times \sum_{s=e, o} \sum_{n=1}^{\infty} \sum_{m=0}^{n} D_{m n}\left[\mathbf{m}_{s m n}^{(3)}\left(\mathbf{r}_{>}\right) \mathbf{m}_{s m n}^{(1)}\left(\mathbf{r}_{<}\right)+\mathbf{n}_{s m n}^{(3)}\left(\mathbf{r}_{>}\right) \mathbf{n}_{s m n}^{(1)}\left(\mathbf{r}_{<}\right)\right] .
\end{aligned}
$$


Application of the conditions (54) simplifies the foregoing expression as follows:

$$
\begin{aligned}
\underline{\underline{G}}\left(\mathbf{r}, \mathbf{r}_{p}\right) & =\frac{i k A^{3}}{\pi} \exp \left[-i k_{0} \boldsymbol{\Gamma} \cdot\left(\mathbf{r}_{>}+\mathbf{r}_{<}-\mathbf{r}+\mathbf{r}_{p}\right)\right] \\
\quad \times \underline{\underline{B}} & \cdot \sum_{s=e, o} \sum_{n=1}^{\infty} \sum_{m=0}^{n} D_{m n}\left[\mathcal{M}_{s m n}^{(3)}\left(\mathbf{r}_{>}\right) \mathcal{M}_{s m n}^{(1)}\left(\mathbf{r}_{<}\right)+\mathcal{N}_{s m n}^{(3)}\left(\mathbf{r}_{>}\right) \mathcal{N}_{s m n}^{(1)}\left(\mathbf{r}_{<}\right)\right] \cdot \underline{\underline{B}}
\end{aligned}
$$

Equations (130) and (131) will be helpful for analyzing aperture antennas [33,34] and radomes made of the chosen material $[35,36]$.

\section{Concluding remarks}

The derivation, in closed-form, of vector spherical wavefunctions for an orthorhombic dielectric-magnetic material with gyrotropic-like magnetoelectric properties, enables the electromagnetic scattering response of an object made of such a material to be comprehensively and conveniently characterized. In particular, these wavefunctions provide the building blocks for (i) the T-matrix formulation which yields a description of the field scattered by a 3D object composed of the chosen material, and (ii) the dyadic Green function which yields a description of the radiation field of a source current density distribution embedded in the chosen material.

Acknowledgments. AL thanks Craig F. Bohren for a discussion regarding the antecedents of the 1908 paper of Mie [6], and the Charles Godfrey Binder Endowment at Penn State for ongoing support of his research activities.

\section{References}

[1] N.A. Logan, "Survey of some early studies of the scattering of plane waves by a sphere," Proc IEEE, 53 (8), 773-785 (1965)

[2] M.T. Prinkey, A. Lakhtakia and B. Shanker, "On the extended Maxwell-Garnett and the extended Bruggeman approaches for dielectric-in-dielectric composites," Optik, 96 (1), 25-30 (1994)

[3] L.V. Lorenz, "Lysvevægelsen i og uden for en af plane lysbølger belyst kugle," K Dan Vidensk Selsk Forh, 6 (6), 1-62 (1890). The significance of this paper is discussed in Refs. [1] and [5].

[4] L.V. Lorenz, "Sur la lumière réfléchie et réfractee par une sphère transparente," in Oeuvres Scientifiques de L. Lorenz, Revues et annotées par H. Valentine, Vol. 1, Libraire Lehmann et Stage, Copenhagen (1898); pp. 404-502. This is a French translation of Ref. [3]. The translator has provided 27 pages of comments after the translated paper.

[5] H. Kragh, "Ludvig Lorenz and nineteenth century optical theory: the work of a great Danish scientist," Appl Opt, 30 (33), 4688-4695 (1991)

[6] G. Mie, "Beiträge zur Optik trüber Medien, speziell kolloidaler Metallösungen," Ann Phys Lpz, 25 (3), 377-445 (1908)

[7] J.A. Stratton, Electromagnetic Theory, McGraw-Hill (1941); Chap. 7

[8] C.F. Bohren and D.R. Huffman, Absorption and Scattering of Light by Small Particles, Wiley (1983); Chap. 4 
[9] C.F. Bohren, "Light scattering by an optically active sphere," Chem Phys Lett, 29 (3), 458-462 (1974)

[10] A. Lakhtakia, Beltrami Fields in Chiral Media, World Scientific (1994)

[11] T.G. Mackay and A. Lakhtakia, Electromagnetic Anisotropy and Bianisotropy, World Scientific (2010); Chap. 4

[12] A. Boström, G. Kristensson and S. Ström, "Transformation properties of plane, spherical and cylidrical scalar and vector wave functions," in: V.V. Varadan, A. Lakhtakia and V.K. Varadan (Eds), Field Representations and Introduction to Scattering, North-Holland (1991); Chap. 4

[13] W. Ren, "Contributions to the electromagnetic wave theory of bounded homogeneous anisotropic media," Phys Rev E, 47 (1), 664-673 (1993)

[14] Y.-L. Geng, X.-B. Wu, L.-W. Li and B.-R. Guan, "Mie scattering by a uniaxial anisotropic sphere," Phys Rev E, 70 (5), 056609 (2004)

[15] A. Lakhtakia and T.G. Mackay, "Dyadic Green function for an electromagnetic medium inspired by general relativity," Chinese Phys Lett, 23 (4), 832-833 (2006). Erratum: 29 (1), 019902 (2012)

[16] T. Danov and T. Melamed, "Spectral analysis of relativistic dyadic Green's function of a moving dielectric-magnetic medium," IEEE Trans Antennas Propagat, 59 (8), 2973-2979 (2011)

[17] A. Lakhtakia and W. S. Weiglhofer, "On electromagnetic fields in a linear medium with gyrotropiclike magnetoelectric properties," Microw Opt Technol Lett, 15 (3), 168-170 (1997)

[18] A. Gray, Modern Differential Geometry of Curves and Surfaces, CRC Press (1993); p. 105.

[19] A. Lakhtakia and W.S. Weiglhofer, "Time-harmonic electromagnetic fields in source regions in a simple uniaxial bianisotropic medium," Int J Appl Electromag Mater, 5 (2), 101-108 (1994).

[20] P.M. Morse and H. Feshbach, Methods of Theoretical Physics, Part II, McGraw-Hill (1953); Chap. 13

[21] K. Aydin and A. Hizal, "On the completeness of the spherical vector wave functions," J Math Anal Appl, 117 (2), 428-440 (1986)

[22] P.W. Barber and C. Yeh, "Scattering of electromagnetic waves by arbitrarily shaped dielectric bodies," Appl Opt, 14 (12), 2864-2872 (1975)

[23] A. Lakhtakia, V.K. Varadan and V.V. Varadan, "Scattering and absorption characteristics of lossy dielectric, chiral, nonspherical objects," Appl Opt, 24 (23), 4146-4154 (1985)

[24] A. Lakhtakia, "On the Huygens's principles and the Ewald-Oseen extinction theorems for, and the scattering of, Beltrami fields," Optik, 91 (1), 35-40 (1992).

[25] H.C. Chen, Theory of Electromagnetic Waves, McGraw-Hill (1983); Chap. 9

[26] A.D. Yaghjian, "Electric dyadic Green's functions in the source region," Proc IEEE, 68 (2), 2488-263 (1980)

[27] A. Lakhtakia, B. Michel and W.S. Weiglhofer, "Field induced in an electrically small dielectric particle embedded in a uniaxial dielectric host medium," Arch Elektron Übertrag, 51 (3), 231-232 (1997).

[28] J.A. Osborn, "Demagnetizing factors of the general ellipsoid," Phys Rev, 67 (11-12), 351-357 (1945)

[29] E.C. Stoner, "The demagnetizing factors for ellipsoids," Phil Mag, 36, 803-821 (1945) 
[30] B. Michel and W.S. Weiglhofer, "Pointwise singularity of dyadic Green function in a general bianisotropic medium," Arch Elektron Übertrag, 51 (3), 219-223 (1997). Erratum: 52 (1), 310 (1998)

[31] W.S. Weiglhofer, "Electromagnetic depolarization dyadics and elliptic integrals," J Phys A: Math Gen, 31 (34), 7191-7196 (1998)

[32] W.S. Weiglhofer, A. Lakhtakia and B. Michel, "Maxwell Garnett and Bruggeman formalisms for a particulate composite with bianisotropic host medium," Microw Opt Technol Lett, 15 (4), 263-266 (1997). Erratum: 22 (3), 221 (1999)

[33] P.J. Wood, "Spherical waves in antenna problems," Marconi Rev, 34, 149-172 (1971)

[34] A. Lakhtakia and M.F. Iskander, "Scattering and absorption characteristics of lossy dielectric objects exposed to the near fields of aperture sources," IEEE Trans Antennas Propagat, 31 (1), 111-120 (1983)

[35] N. Engheta and M.W. Kowarz, "Antenna radiation in the presence of a chiral sphere," J Appl Phys, 67 (2), 639-647 (1990)

[36] A. Lakhtakia, V.K. Varadan and V.V. Varadan, "Radiation by a point electric dipole embedded in a chiral sphere," J Phys D: Appl Phys, 23 (5), 481-485 (1990) 